\title{
Um método robusto de segmentação de imagem de íris
}

\author{
Daniel Felix de Brito e Lee Luan Ling
}

\begin{abstract}
Resumo: Este artigo apresenta um método robusto para localização rápida da pupila, íris e das pálpebras. $O$ método tem como objetivo incrementar o desempenho de sistemas de reconhecimento biométrico de íris através da redução do tempo de processamento na etapa de segmentação de imagem de íris.
\end{abstract}

Palavras Chaves: Segmentação de Imagem de Íris, Reconhecimento de Íris, Biometria.

Abstract - This paper presents an algorithm for fast localization of iris image, increasing the performance of iris biometric recognition systems by reducing the processing time during the phase of iris image segmentation.

Index Terms - Iris Image Segmentation, Iris Recognition, Biometrics.

\section{INTRODUÇÃO}

O termo biometria refere-se, no contexto deste trabalho, à identificação de um individuo baseada em suas características físicas ou comportamentais. O sucesso da tecnologia biométrica deve-se à obrigatoriedade da presença física do individuo no local de identificação e à eliminação da necessidade de memorizar senhas ou transportar identificadores que poderiam ser facilmente esquecidos ou roubados.

Em termos da biometria de íris, várias abordagens têm sido desenvolvidas nos últimos anos, dentre as quais destacam-se os métodos relatados nos trabalhos de J. Daugman [1] e Y. Du [6]. A primeira etapa de um processo reconhecimento de íris consiste da captura da imagem de olho e melhoramento da imagem adquirida. Em seguida, a imagem melhorada é segmentada para que a região da íris possa ser delineada. Uma boa revisão dos principais métodos de segmentação da região de íris, amplamente usados atualmente, pode ser encontrada em [4]. Muitos destes métodos, embora altamente eficientes na localização e extração da região de íris, são em geral altamente custosos computacionalmente, comprometendo sua aplicações em tempo real.

Daniel Felix de Brito e Lee Luan Ling, Departamento de Comunicação da Faculdade Engenharia Elétrica e Computação, Universidade Estadual de Campinas - UNICAMP, Brasil. E-mails: fbrito.daniel@gmail.com, lee@decom.fee.unicamp.br,
Em [7], Chavez apresenta comparativamente os tempos médios de processamento por estes métodos na segmentação de imagem de íris.

Este trabalho tem como objetivo desenvolver novas abordagens de segmentação de imagem de íris de modo a manter a qualidade do resultado da segmentação, porém com tempos de execução aceitável para reconhecimento de Íris em tempo real.

\section{SEGMENTAÇÃO - MÉTODOS}

O processo de segmentação de imagem de íris aqui proposto pode ser dividido em 3 etapas:

- $\quad$ Detecção de pupila;

- Delineamento de bordas laterais de íris;

- Localização de pálpebra inferior e pálpebra superior.

A grande vantagem do método apresentado consiste em que a etapa seguinte é efetuada levando em consideração os resultados da etapa anterior. Desde modo, conseguimos eliminar grande parte do processamento de imagem de regiões desnecessárias para segmentar a imagem.

\section{A. Detecção de Pupila}

A abordagem para detectar a pupila pode ser dividida em 5 passos:

- Binarização da imagem;

- Determinação das sub-regiões da imagem;

- Delimitação da sub-região relevante;

- Redução dos efeitos dos ruídos (reflexos);

- Detecção de borda (determinação de centro e raio).

\section{A.l Binarização da Imagem}

Após aquisição de imagem original, a partir de um leitor ótico ou amostra do banco de dados, aplicamos um filtro Gaussiano para melhorá-la. Chamamos a imagem resultante de imagem melhorada.

O primeiro passo da detecção de pupila no algoritmo proposto é a binarização da imagem melhorada. Este passo tem como objetivo separar os objetos de interesse da imagem do fundo. $\mathrm{O}$ valor do limiar utilizado para gerar uma imagem binária é determinado previamente da análise sobre o histograma dos níveis de cinza. Essa análise é realizada de forma automática. $\mathrm{Na}$ 
escala cinza de 256 níveis $(0-255)$, faça $S$ a quantidade de pixels presentes na imagem com nível de cinza variando de 0 a 150 . O limiar de binarização será o nível cuja soma do total de pixels dos níveis menores resulte em $70 \%$ do valor de $\mathrm{S}$.

O valor $70 \%$ foi escolhido após um estudo sobre os resultados obtidos variando o limiar no intervalo $50-100 \%$ com objetivo de preservar o máximo possível à integridade da região da pupila. Assim, os valores abaixo do limiar terão seu valor alterado para 0 (preto) e serão considerados como pertencentes a algum objeto e acima para 255 (branco) e serão considerados como pixels de fundo. $\mathrm{O}$ intervalo para calcular $\mathrm{S}$ foi definido de $0-150$ devido às características da pupila. Observando a pupila verificamos que não possui pixels com valor de brilho maior do que 150 .

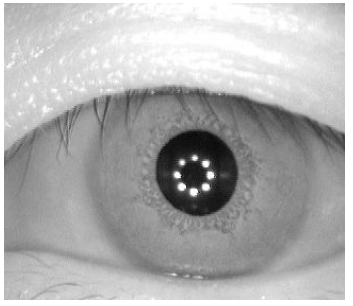

( a )

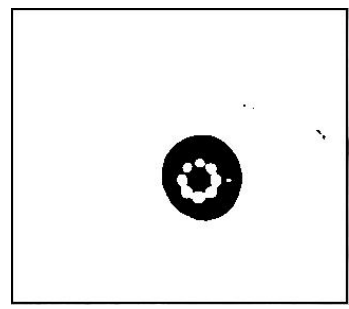

(c)

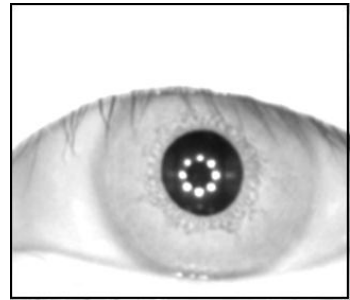

( b )

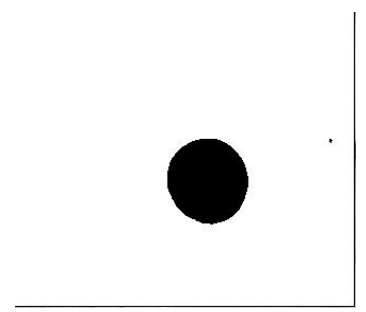

( d )

\begin{tabular}{|l|l|l|l|l|}
\hline $1.3 / 10.7$ & $3.2 / 10.7$ & $3.8 / 10.7$ & $3.2 / 10.7$ & $1.3 / 10.7$ \\
\hline
\end{tabular}

\begin{tabular}{|l|}
\hline $1.3 / 10.7$ \\
\hline $3.2 / 10.7$ \\
\hline $3.8 / 10.7$ \\
\hline $3.2 / 10.7$ \\
\hline $1.3 / 10.7$ \\
\hline
\end{tabular}

(e)

$$
\left[\begin{array}{rrr}
1 & 2 & 1 \\
0 & 0 & 0 \\
-1 & -2 & -1
\end{array}\right]
$$

Gy

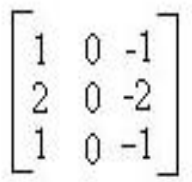

Gx (f)

Fig. 1: (a) Imagem original. (b) Imagem binária: limiar igual a 100. ( c ) Após detecção de ruído (reflexo dentro da pupila). (d) Após do filtro gaussiano. ( e ) Filtro gaussiano: direções X (horizontal) e Y (vertical) (f) operadores de Sobel
A figura 1-a ilustra uma imagem original. As figuras 1-b, 1-c, 1-d e 1-e ilustram, respectivamente: imagem melhorada, imagem binária após aplicar limiar, imagem após determinar ruído dentro da pupila e filtro gaussiano aplicado para melhorar a imagem original.

\section{A.2 Determinação das sub-regiões da imagem}

Nesta etapa, a imagem binarizada será dividida em sub-regiões para que possamos determinar aquela sub-região que contém a pupila. Uma sub-região, aqui definida, é composta de um conjunto de pixels adjacentes com valor zero (pretos), sendo que cada pixel desse conjunto possui seus 8 vizinhos também com o valor zero. Assim, a imagem da figura 1-c possui uma sub-região maior no centro e diversas pequenas sub-regiões à sua volta.

\section{A.3 Delimitação da sub-região relevante}

A sub-região que possui a maior quantidade de pixels com valor zero (preto) será a região de análise, para confirmação ou não da região que contém ou forma a pupila. As sub-regiões restantes na imagem binária terão seus pixels com valores alterado de 0 para 255, passando a serem considerados como pixels de fundo.

\section{A.4 Detecção da borda (determinação do centro e raio)}

Assumimos que a borda de uma pupila é modelada por uma circunferência com centro $\left(\mathrm{X}_{\mathrm{p}}, \mathrm{Y}_{\mathrm{p}}\right)$ e o raio $\left(\mathrm{R}_{\mathrm{p}}\right)$. Assim sendo, o método apresentado tem como objetivo estimar estes parâmetros. Para estimar os parâmetros, usamos a imagem binária com a subregião delimitada da etapa anterior.

Inicialmente efetuamos o realce de bordas da sub-região delimitada na imagem binária. A técnica adotada nesta utiliza os vetores gradientes extraídos da imagem. Os vetores gradientes são obtidos através da aplicação dos operadores de Sobel, ilustrados na figura 1-f, para encontrar o módulo e o ângulo do gradiente de cada pixel em analise. A figura 2-b ilustra a subregião com borda realçada.

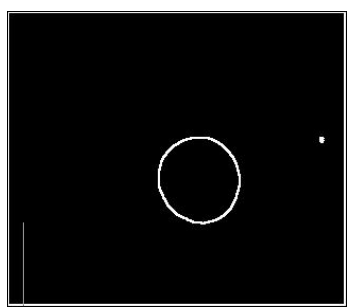

Fig. 2: borda da pupila realçada

Os pixels da borda realçada são em seguida agrupados de acordo com seus respectivos ângulos gradientes. Neste trabalho agrupamos em 4 conjuntos. Cada conjunto representa um dos quatro quadrantes. Assim, se um pixel possuir valor de ângulo gradiente entre zero e 90, será adicionado ao primeiro conjunto, 
logo pertencendo ao primeiro quadrante. E assim sucessivamente para outros 3 conjuntos, os correspondentes quadrantes.

A determinação dos parâmetros $\left(X_{p}, Y_{p}\right)$ e $R_{p}$ consiste em detectar dois pontos das bordas de quadrantes opostos que distanciam exatamente o valor do diâmetro. Conseqüentemente, $o$ ponto médio destes dois pontos das bordas detectados especifica a posição do centro da circunferência. Operacionalmente combinamos a metade dos pixels na borda em quadrantes opostos para simultaneamente acelerar o processo estimação e minimizar $o$ erro. Assim sendo, o centro $\left(X_{p}, Y_{p}\right)$ da circunferência é dado pelo pixel com a maior freqüência de ocorrência como sendo o ponto médio entre dois pontos da borda combinados. Analogamente, o raio $\mathrm{R}_{\mathrm{p}}$ é dado pelo valor mais provável.

Note que, para estimar (Xp, Yp), utilizamos o conceito de células acumuladoras de Hough, mas não utilizamos as fórmulas matemáticas de circunferência, o que implica um ganho significativo no tempo de processamento.

O método proposto leva em consideração que a pupila pode sofrer dilatação.

\section{A.5. Redução dos efeitos dos ruídos (reflexos)}

$\mathrm{O}$ método de detecção de borda poderia não funcionar corretamente caso houvesse presença significativa de ruído devido ao reflexos na região interior da pupila. Assim, após a seleção da sub-regiões relevantes, antes da detecção de borda, teríamos que eliminar estes ruídos presentes. Para isto, procuramos inicialmente identificar cada região de reflexo dentro da área da pupila. Um ponto (pixel) será considerado como ruído, caso seu valor de pixel seja o valor de fundo e em cada uma das direções (horizontal e vertical) possua pelo menos um ponto (pixel) apresentado valor zero (preto). A figura 3 ilustra o procedimento envolvido onde pixels envolvidos. $\mathrm{O}$ pixel em análise é destacado em vermelho. Assim, seus vizinhos nas direções horizontal e vertical são analisados. Caso exista pelo menos um pixel em cada uma das direções com valor 0 , o pixel em análise terá seu valor alterado para 0 . Neste caso, este deixa de ser fundo (255) e passa a ser objeto (0).

\begin{tabular}{|c|c|c|c|c|}
\hline & & \multirow{2}{*}{$:$} & & \\
\hline & & & & \\
\hline & & $\mathrm{x}$ & & \\
\hline$\cdots$ & $\mathrm{x}$ & pixe & $\mathrm{x}$ & $\cdots$ \\
\hline & & $\mathrm{x}$ & & \\
\hline & & \multirow{2}{*}{$:$} & & \\
\hline & & & & \\
\hline
\end{tabular}

Fig. 3: No centro em vermelho temos o pixel em análise. Em cada direção os pixels que serão verificados se são objeto ou não.

Todos os pixels da imagem binária da sub-região selecionada são analisados. A figura 1-d ilustra o resultado do processamento feito na figura 1-c, onde o ruído (reflexos) dentro da pupila foi detectado e eliminado corretamente.

\section{B. Segmentação de Íris}

O processo de detecção da íris implementado neste trabalho pode ser dividido em 4 passos:

- Delimitação da região de interesse;

- Identificação do melhor filtro mediano;

- Determinação da borda da íris;

- Verificação da borda da íris.

Assumimos que a borda de uma íris é modelada por uma circunferência com centro $\left(\mathrm{X}_{\mathrm{i}}, \mathrm{Y}_{\mathrm{i}}\right)$ e o raio $\left(\mathrm{R}_{\mathrm{i}}\right)$. Assim sendo, o método apresentado tem como objetivo estimar estes parâmetros.

Um problema a ser resolvido em detecção da borda da íris é que nem sempre a imagem de olho adquirida possui um contraste claro entre a íris e a esclerótica (zona branca do globo ocular). O algoritmo aqui proposto leva em consideração este fator. Note que, em geral, a pupila e a íris não são objetos concêntricos. Em outras palavras, o centro da pupila não coincide com o centro do anel de íris.

\section{B.1 Delimitação da região de interesse}

Inicialmente, geramos uma nova imagem binária a partir da imagem melhorada, aplicando a técnica de gradiente na imagem. O limiar será o valor de gradiente médio.

A região de interesse será a menor parte inferior da nova imagem que contém a região da pupila. Neste trabalho, determinamos esta área utilizando os parâmetros encontrados $Y_{p}$ e $R_{p}$. Em outras palavras, traçamos uma linha horizontal passando pelo ponto $\left(\mathrm{X}_{\mathrm{p}}, \mathrm{Y}_{\mathrm{p}}+\mathrm{Rp}\right)$. As figuras 4-a e 4-b ilustram a linha traçada e a região de interesse delimitada.

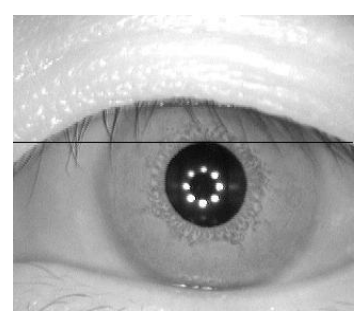

( a )

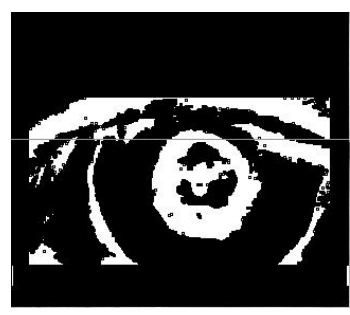

( b )
Fig. 4: Linha delimitadora (a) Imagem original; (b) Imagem binária

\section{B.2 Identificação do melhor filtro mediano}

O objetivo desta etapa é eliminar a textura da íris através de um filtro mediano adequado, facilitando o trabalho de realce das bordas da área de íris. O filtro mediano é encontrado através de um processo interativo envolvendo os passos de detecção e verificação de borda a seguir. Em outras palavras, variamos o tamanho $n_{\mathrm{x}} n$ do tamanho da máscara do filtro até o resultado satisfatória de eliminação de textura de íris ser obtido. 
Operacionalmente, para cada pixel $\mathrm{p}$ da imagem, ordenamos seu valor junto aos valores dos $n^{2}$ pixels vizinhos ao redor. Neste trabalho, $\mathrm{n}$ assume um valor do intervalo $10 \leq n \leq 55$. Começamos com um filtro mediano de tamanho 10x10 $(n=10)$ na região de interesse. Se a borda de íris não for detectada, aumentamos o valor de $\mathrm{n}$ até 15 , e repetimos o processo de detecção (vide as subseção a seguir). Este processo de procurar é repetido até 6 vezes sempre incrementando n por 5. Note que as filtragens são sempre realizadas utilizando os valores de brilho da mesma imagem original. Apesar de, na pior das hipóteses, utilizarmos 6 filtros medianos sobre a imagem, note que utilizamos apenas sobre um pequeno conjunto de pixels e não sobre toda a imagem. Devido a isto, o desempenho em termos de velocidade não é comprometido, ainda assim apresentando um baixo custo computacional.

\section{B.3 Determinação da borda da íris}

Este passo consiste em determinar o centro da circunferência que modela a íris $\left(\mathrm{X}_{\mathrm{i}}, \mathrm{Y}_{\mathrm{i}}\right)$ e o raio $\left(\mathrm{R}_{\mathrm{i}}\right)$. Para isto, realçamos as bordas da região de interesse utilizando a técnica do vetor gradiente e utilizamos a mesma técnica usada na detecção da pupila. Porém, devido a maior incerteza existente em virtude do baixo contraste na região de borda de íris, apenas aqueles pixels que estiverem suas distâncias próximas ao centro da pupila são usados. Por exemplo, temos duas coordenadas de borda (valor 255) como candidatas $\left(\mathrm{x}_{1}, \mathrm{y}_{1}\right)$ e $\left(\mathrm{x}_{2}, \mathrm{y}_{2}\right)$. Se a distância de $\left(\mathrm{x}_{1}, \mathrm{y}_{1}\right)$ ao $\left(X_{p}, Y_{p}\right)$ for $95 \%$ próxima da distancia entre $\left(x_{2}, y_{2}\right)$ e $\left(X_{p}, Y_{p}\right)$, efetuamos a aproximação de $\left(\mathrm{X}_{\mathrm{i}}, \mathrm{Y}_{\mathrm{i}}\right)$ utilizando ambos os pontos. Caso contrário, os pontos são descartados. Esse procedimento é fundamental para eliminação de ruídos. A figura 5 ilustra exemplos das fronteiras da pupila e da íris encontradas.

\section{B.4 Verificação da borda da íris encontrada}

Este passo tem como objetivo verificar a estimativa dos parâmetros $\left(\mathrm{X}_{\mathrm{i}}, \mathrm{Y}_{\mathrm{i}}\right)$ e $\mathrm{R}_{\mathrm{i}}$. Uma vez encontrada a posição aproximada do centro e o valor estimado do raio, construímos uma circunferência com estes parâmetros e verificamos a quantidade de pixels da borda sobrepõe sobre a circunferência. A borda de íris é declarada detectada, quando há mais de $30 \%$ de sobreposição. Caso contrário, o próximo valor de n é testado.

Os testes experimentais realizados indicam que normalmente no máximo apenas três valores de $\mathrm{n}$ são testados. Note que o valor limiar de $30 \%$ de sobreposições foi determinado empiricamente, e comprovado eficiente.

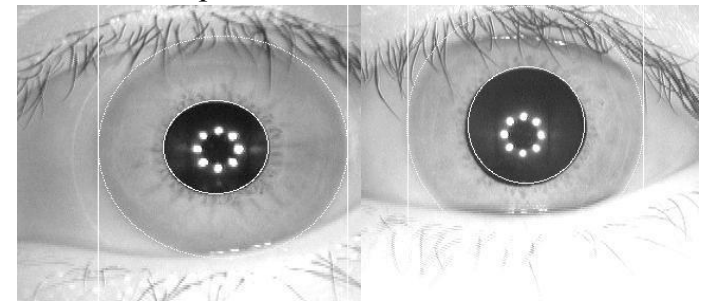

Fig. 5: Exemplos de da localização das fronteiras da íris e da pupila

\section{Detecção das pálpebras}

Esta sub-seção tem como objetivo descrever o procedimento de determinação de pálpebras superior e inferior. Este procedimento é necessário, pois como podemos observar nas Figuras 5 e 6, parte da íris pode ser sobreposta pelas pálpebras. Assim, na etapa de normalização, que será explicado neste artigo, ao amostrarmos pontos da íris devemos desconsiderar ponto que estão acima da parte superior e abaixo da inferior.

Podemos dividir este processo de detecção das pálpebras em 2 passos, nominalmente:

- Delimitar a região de interesse;

- Encontrar os pixels de borda dentro da região e estimar região abaixo da pálpebra inferior e acima da pálpebra superior;

Como dito anteriormente, a vantagem de encontrar a pupila e a íris primeiro, em um processo de segmentação de íris, é a redução considerável da área de busca na detecção de pálpebras. Para determinar precisamente a localização da pálpebra inferior, geramos uma janela retangular em torno de uma região considerada mais provável relativa à localização da pupila e da íris. As coordenadas dos quatro vértices da janela retangular são obtidas da seguinte forma. Sejam

$$
\begin{aligned}
& \mathrm{x}_{\mathrm{i}}=\mathrm{X}_{\mathrm{i}}-\mathrm{R}_{\mathrm{i}} ; \\
& \mathrm{y}_{\mathrm{i}}=\mathrm{Y}_{\mathrm{p}}+\mathrm{R}_{\mathrm{p}} ; \\
& \mathrm{x}_{\mathrm{s}}=\mathrm{X}_{\mathrm{i}}+\mathrm{R}_{\mathrm{i}} ; \\
& \mathrm{y}_{\mathrm{s}}=\mathrm{l}_{\text {max. }}
\end{aligned}
$$

onde $1_{\max }$ é o número de linhas da imagem e:

- $\quad\left(\mathrm{x}_{\mathrm{i}}, \mathrm{y}_{\mathrm{i}}\right)$ a coordenada superior-esquerda;

- $\quad\left(\mathrm{x}_{\mathrm{s}}, \mathrm{y}_{\mathrm{i}}\right)$ a coordenada superior-direita;

- $\quad\left(\mathrm{x}_{\mathrm{i}}, \mathrm{y}_{\mathrm{s}}\right)$ a coordenada inferior-esquerda;

- $\quad\left(\mathrm{x}_{\mathrm{s}}, \mathrm{y}_{\mathrm{s}}\right)$ a coordenada inferior-direita;

- $\quad\left(\mathrm{X}_{\mathrm{i}}, \mathrm{Y}_{\mathrm{i}}\right)$ : coordenadas do centro da íris e $\mathrm{R}_{\mathrm{i}}$ seu raio;

- $\quad\left(X_{p}, Y_{p}\right)$ : coordenada do centro da pupila e $R_{p}$ o raio.

A figura 6 ilustra um exemplo da janela retangular encontrada em um imagem de olho.

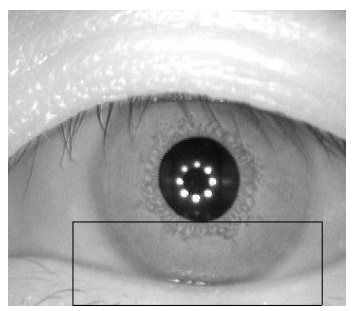

Fig. 6: Região de interesse para encontrar a pupila

Para localizar as pálpebras, utilizamos a mesma imagem binária utilizada para detectar a íris. 
A idéia básica de detecção de pálpebra consiste em varrer as colunas da janela e determinar um pixel de cada coluna que faz parte da pálpebra. Pode ocorrer, devido ao ruído, que em algumas colunas os pixels de pálpebra não são detectados. Neste caso, o valor do pixel de borda desta coluna será estimado através dos valores dos pixels de seus vizinhos laterais. Em outras palavras, o valor do pixel considerado como pertencente à pupila, na coluna corrente, será o pixel cuja linha for o resultado da média das linhas dos dois pixels vizinhos mais próximos classificados como pertencentes à pupila. A figura 7 mostra dois exemplos do resultado do processo de detecção de pálpebra, porém, Para fins ilustrativos, usamos imagens originais.

O mesmo procedimento é aplicado para encontrar a borda da pálpebra superior, alterando apenas as coordenadas limites da janela. A figura 7 ilustra a pálpebra superior encontrada. Analisando as figuras 6 e 7 observamos que apesar da grande presença de cílios, a pálpebra superior foi encontrada corretamente. Observa-se também a presença de cílios sobre a íris. Alguns testes que efetuamos demonstram que os cílios não interferem de maneira significativa no resultado final do reconhecimento. Isto faz com que alguns autores não eliminam os cílios e que não há necessidade de se preocupar com essa interferência, considerada como ruído.

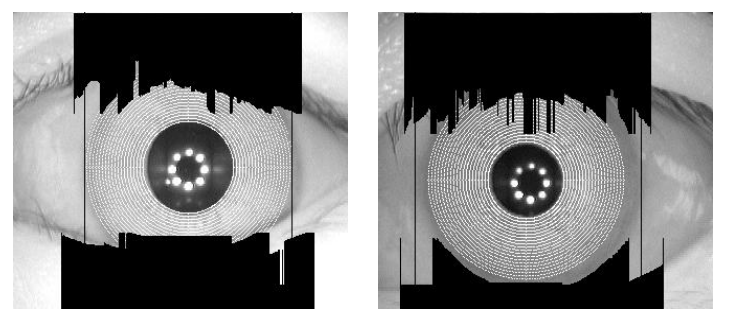

Fig. 7: Exemplos de localização das pálpebras e os anéis da íris

\section{NORMALIZAÇÃO}

Variação nas condições de captura da imagem da íris provoca variações na dilatação da pupila, na área da íris ou na concentricidade entre a íris e a pupila. $\mathrm{O}$ algoritmo de normalização proposto tem como objetivo minimizar esses efeitos. Para isto, a área que contém a textura de íris é dividida em 20 anéis distintos e concêntricos. Cada anel é dividido em 180 partes. Assim, são amostrados 3600 valores da imagem original, construindo a imagem normalizada. Na figura 7 temos uma ilustração dos anéis formados. Mais detalhes sobre o processo de normalização pode ser consultado em [4].

Note que como pode ocorrer que as circunferências da borda da pupila e do Íris não são concêntricas, é necessário garantir que os pontos usados para formação de anéis não sejam pontos de pupila e de pontos não pertencentes à área de Íris. Este procedimento de verificação é de fundamental importância, visto que esses pontos externos podem causar uma degradação considerável do desempenho do sistema de reconhecimento caso eles não são eliminados.

\section{FILTRO GABOR E CONVOLUÇÃO}

Nesta etapa, a imagem normalizada é processada e informações relevantes são extraídas para uma fim de comparação/verificação. Forma que permite uma comparação rápida. Basicamente dois procedimentos são envolvidos nesta etapa: transformação rápida de Fourier sobre a imagem normalizada e convolução entre a imagem resultante e um filtro de Gabor. Os resultados das operações envolvidas nesta etapa é um vetor binário, muitas vezes é conhecido com o código de Íris. Os maiores detalhes sobre estes dois procedimentos podem ser encontrada em [1] e [4].

\section{ARMAZENAMENTO E COMPARAÇÃO}

Par fim de testes, cada vetor binário obtido na etapa anterior é salvo em um arquivo teste. Deste modo, gera-se para cada imagem de cada indivíduo uma representação binária de sua íris, sendo esta armazenada. Posteriormente, estes dados são lidos, armazenado em vetores e comparados. Para comparação utilizamos a distância de Hamming. Este método fornece um índice que indica o quanto dois vetores binários são diferentes. Para mais detalhes, consulte [4].

\section{ESTUDO EXPERIMENTAL E CONCLUSÃO}

Os algoritmos propostos foram testados por um conjunto de 2400 imagens do banco de dados [9]. Os testes experimentais revelam que a menor taxa de acertos para a correta segmentação da pupila e da íris foi de $97 \%$, sendo $98 \%$ para a pupila e $97 \%$ para a íris. Além disso, o tempo total de detecção para cada imagem não ultrapassou 4 segundos. Utilizando o mesmo banco de dados e as mesmas condições de hardware, rodamos os algoritmos de detecção de íris, pupila e pálpebras disponibilizados por Libor Masek [4]. Em relação ao tempo de processamento, nosso algoritmo fornece um ganho de 15 vezes em relação ao de Masek. Se compararmos em taxas de acertos, na etapa de segmentação, temos um ganho de $10 \%$ em relação a Masek [4], mantendo ao final de todas as etapas do processo de reconhecimento praticamente as mesmas taxas de detecção (FRR e FAR), sendo inferior a $1 \%$ se descartarmos as imagens não segmentadas corretamente e $12 \%$ caso não sejam descartadas. Daugman [1], o principal autor na área, em diversos artigos fornece os erros FRR e FAR inferiores a $1 \%$, descartando as imagens não segmentadas corretamente. Conforme mencionado por [10] é de difícil comparação a etapa de segmentação com a utilizada por Daugman, pois este não entra em detalhes sobre seu método. Porém, a taxa média de acertos de Daugman para esta etapa está em torno de $60 \%$. Comparando com esta taxa, nosso algoritmo apresenta um alto ganho em número de acertos, mantendo a taxa final de reconhecimento abaixo de 1\% (ao descartar as imagens segmentadas incorretamente). Assim, nossos algoritmos melhoram a etapa de segmentação, o que traz a vantagem de ser necessário um número menor de captura de imagens para fins comparativos no reconhecimento. E ainda 
assim, mantêm as taxas de reconhecimentos apresentadas pelos principais autores na área.

A figura 8 apresenta a curva ROC obtida com os resultados ao final do processo de reconhecimento considerando as imagens não detectadas corretamente na etapa de segmentação. A figura 9 apresenta a curva ROC desconsiderando as imagens segmentadas incorretamente. Comparando ambas as curvas, verificamos como a etapa de segmentação é importante no processo todo, pois uma imagem não segmentada corretamente pode levar há um erro no processo final de reconhecimento. A figura 9 mostra que ao descartar as imagens não segmentadas corretamente, mantivemos as taxas de erros apresentadas na literatura. Dessa forma, como nosso projeto obtém um ganho na etapa de segmentação, através do descarte de uma quantidade menor de imagens, o que o torna melhor do que os sistemas apresentados na literatura. Além disso, temos o fator do tempo de execução, uma vez que nosso método para segmentar é mais rápido do que os apresentados na literatura, como discutido.

A correta segmentação é fundamental para o processo de reconhecimento. Com a nova metodologia proposta, além de reduzirmos os tempos de segmentação, aumentados a taxa de acertos nesta etapa, mantendo a alta precisão das etapas seguintes no reconhecimento. Com isto, podemos concluir que nosso algoritmo de deteç̧ão de íris é mais apropriado para ser utilizado em sistema de Reconhecimento de íris, podendo vir a substituir os métodos atuais.

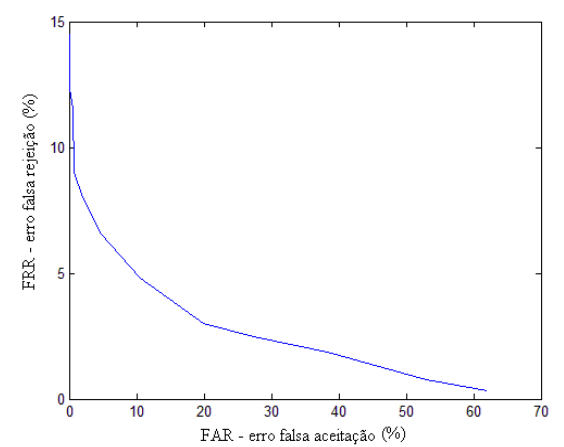

Fig. 8: curva ROC considerando imagens não segmentadas corretamente

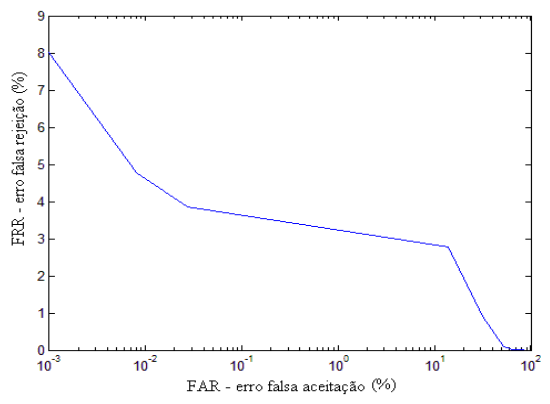

Fig. 9: curva ROC descartando imagens não segmentadas corretamente

\section{REFERÊNCIAS}

[1] J. G. Daugman, "How Iris Recognition Works" IEEE Trans. On Circuits and Systems for Video Technology, vol. 14, n. 1, pp. 21-30, 2004.

[2] J. Daugman, "Probing the uniqueness and randomness of IrisCodes: Results from 200 billion iris pair comparisons." Proceedings of the IEEE, vol. 94, no. 11, pp 1927-1935, 2006

[3] ICE 2006 Report: P. Jonathon Phillips, W. Todd Scruggs, Alice J. O’Toole, Patrick J. Flynn, Kevin W. Bowyer, Cathy L. Schott, and Matthew Sharpe. "FRVT 2006 and ICE 2006 Large-Scale Results." NISTIR 7408, March 2007.

[4] L. Masek, "Recognition of Human Íris PAtterns for Biometric Identification", bacharelado, Dept. The University of Western, Australia, 2003

[5] R. C. Gonzáles e R. E. Woods, "Processamento de Imagens Digitais", ed. Edgard Blücher, 2000.

[6]Y. Du, B. Bonney, R. W. Ives, D. M. Etter, e R. Schultz, "Partial Iris Recognition Using a 1-D mApproach: Statistics and Analysis," presented at the 2005 IEEE International Conference on Acoustics, Speech and Signal Processing (ICASSP), Philadelphia, PA, Mar 2005.

[7] R. F. L. Chavez, "Uma Proposta para Melhoria na Eficiência de um Sistema de Reconhecimento de Íris Humana", dissertação de mestrado, Engenharia Elétrica e Computação, Dept. Comunicações, Universidade Estadual de Campinas, São Paulo, 2007.

[8] R. Wildes, "Íris Recognition: An Emerging Biometric Techology," Proceedings of the IEEE, vol. 85, pp. 1348-1363, 1997.

[9] CASIA Iris Image DataBbase (ver 3.0), Institute of Automation, Chinese Academy of Sciences.

[10] Roger Fredy Larico Chavez, Yuzo Iano \& Vicente Idalberto B. Sablon , "Processo de Reconhecimento de Íris Humana: localização rápida de íris", Revista Cientifica Periódica - Telecomunicações, Vol. 09, No. 01, Novembro de 2006. 